\title{
ACHIEVEMENTS IN WORKPLACE NEUTRON DOSIMETRY IN THE LAST DECADE: LESSONS LEARNED FROM THE EVIDOS PROJECT
}

\author{
R. J. Tanner 1,*, T. Bolognese-Milsztajn'2, M. Boschung ${ }^{3}$, M. Coeck ${ }^{4}$, G. Curzio ${ }^{5}$, F. d'Errico 5 , A. Fiechtner ${ }^{3}$, \\ J.-E. Lillhök ${ }^{6}$, V. Lacoste ${ }^{2}$, L. Lindborg ${ }^{6}$, M. Luszik-Bhadra ${ }^{7}$, M. Reginatto ${ }^{7}$, H. Schuhmacher ${ }^{7}$ and \\ F. Vanhavere ${ }^{4}$ \\ ${ }^{1}$ Radiation Protection Division, Health Protection Agency, Chilton, Didcot OX11 0RQ, UK \\ ${ }^{2}$ Institut de Radioprotection et de Sûreté Nucléaire, F-92265 Fontenay-aux-Roses, France \\ ${ }^{3}$ Paul Scherrer Institut, CH-5232 Villigen, Switzerland \\ ${ }^{4}$ Studiecentrum voor Kernenergie-Centre d'étude de l'énergie nucléaire, B-2400 Mol, Belgium \\ ${ }^{5}$ Dipartimento di Ingegneria Meccanica, Nucleare e della Produzione, I-56126 Pisa, Italy \\ ${ }^{6}$ Swedish Radiation Protection Authority, SE-171 16 Stockholm, Sweden \\ ${ }^{7}$ Physikalisch-Technische Bundesanstalt, D-38116 Braunschweig, Germany
}

\begin{abstract}
The availability of active neutron personal dosemeters has made real time monitoring of neutron doses possible. This has obvious benefits, but is only of any real assistance if the dose assessments made are of sufficient accuracy and reliability. Preliminary assessments of the performance of active neutron dosemeters can be made in calibration facilities, but these can never replicate the conditions under which the dosemeter is used in the workplace. Consequently, it is necessary to assess their performance in the workplace, which requires the field in the workplace to be fully characterised in terms of the energy and direction dependence of the fluence. This paper presents an overview of developments in workplace neutron dosimetry but concentrates on the outcomes of the EVIDOS project, which has made significant advances in the characterisation of workplace fields and the analysis of dosemeter responses in those fields.
\end{abstract}

\section{INTRODUCTION}

In most workplaces, where neutron dose rates are of concern, the assessment of those doses is complicated by the wide range of energies that dosemeters and instruments are expected to measure. In industries associated with the nuclear fuel sector, this range is at least 10 orders of magnitude, which causes difficulties with the design of any device that is expected to assess doses to workers who are exposed in those fields.

The goal of designing neutron dosemeters and instruments with dose-equivalent response characteristics that are independent of neutron energy and direction remains an elusive one. This is not surprising given the nature of the task, but improvements to the systems that are available continue to be made, the main development of the last $10 \mathrm{y}$ being the greater availability of active neutron personal dosemeters.

Although $10 \mathrm{y}$ ago, active neutron personal dosemeters were commercially available, they were not in widespread use and were of questionable quality ${ }^{(1)}$. Consequently, most neutron personal dosimetry were provided by passive devices that are inherently retrospective in nature.

An earlier European Commission funded project (ANDO) focused on the development of active

*Corresponding author: rick.tanner@hpa-rp.org.uk neutron dosimetry for individual monitoring ${ }^{(2)}$. It looked at a range of designs of active neutron personal dosemeter, none of which, at that stage, were available commercially. Within the project, it was intended that these designs would be developed to working prototypes that could be tested in calibration fields at PTB to verify their suitability for use in the workplace.

At the end of ANDO, there were several designs of active neutron personal dosemeter that had demonstrated promising performance in the calibration laboratory. However, for active devices, the workplace presents a particular challenge because of the energy range of the neutrons and their direction distribution, high photon dose rates and environmental challenges. Passive dosemeters face the same challenges, but they may be expected to be less sensitive to them, especially the photon dose rate, microphonics and electromagnetic fields.

Consequently, the Evaluation of Individual Dosimetry in mixed neutron and photon radiation fields (EVIDOS) project proposed to follow up ANDO by extending the measurement programme to look at the performance of active neutron personal dosemeters in real workplaces. Some of the most promising designs from the ANDO project were included, as well as other devices that were commercially available. 


\section{R. J. TANNER ET AL.}

Determination of the neutron energy distribution via multispheres and recoil counters is well established ${ }^{(3)}$, but the data that result only permit the $H^{*}(10)$ rate to be calculated. However, assessment of the performance of personal dosemeters in the workplace is only meaningful if their readings can be compared to reference values for $H_{\mathrm{p}}(10)$ and effective dose. Additionally, the angle dependence of response of neutron survey instruments affects their reading in the workplace, so knowledge of the direction distribution of the field is necessary to understand their performance.

Since there were no specific instruments available for determining the energy and direction distribution of a neutron field ${ }^{(4)}$ available at the start of the EVIDOS project, much of the effort within the project went towards developing such methods. These distributions were used to determine $H_{\mathrm{p}}(10)$ for the slab phantom, the quantity defined for the calibration of personal dosemeters, and effective dose for different orientations of the phantom and a person, respectively, in these radiation fields. Two systems were proposed for doing this in detail, where the supporting information would be provided by personal dosemeters placed on the faces of phantoms. An additional system for directly measuring personal dose equivalent, without resolving the energy and direction distribution of the field, would also provide reference values for that quantity.

Within the project, reference values for $H^{*}(10)$ were provided by multispheres. These were needed to understand the full implications of the health physics measurements that would be made in a particular workplace by neutron survey instruments.

Measurements with active neutron personal dosemeters were made at the reference positions on ISO slab phantoms. These were augmented by measurements made using passive detectors, including those used at the laboratory hosting the measurements. The readings of these dosemeters were compared with the reference values determined for the field in order to determine the response and thereby assess the performance of the dosemeter in that field.

\section{THE EVIDOS PROJECT}

Seven laboratories collaborated on the EVIDOS project. This group was assembled to give a good blend of expertise in reference dosimetry, personal dosimetry and workplace field analysis. Access to nuclear sites was also an important criterion, since the project would stand or fall on the availability of workplaces in which the dosemeters could be tested.

The project restricted itself to workplaces connected to the nuclear energy programme. Consequently, energies greater than a few $\mathrm{MeV}$ were of no concern. Initially, however, measurements were made in simulated workplace fields at Cadarache, France
$\left(\mathrm{CANEL}^{(5)}\right.$ and SIGMA $\left.{ }^{(6)}\right)$, since these were already well characterised. This gave the first test of the energy and direction spectrometers, but also meant that the active devices could be measured in 'workplace fields' before the energy and direction spectrometers were fully developed.

Four measurement campaigns were conducted in real workplaces.

- Krümmel boiling water reactor (BWR) in Germany: two locations in the reactor and two locations near a spent fuel flask with its neutron shield removed. One of the reactor locations was inside the containment beneath the core, which provided an unusual direction distribution. This was also a demanding location in terms of environmental conditions. Because the fuel flask had its neutron shield removed, the dose rates were relatively high and the fields were quite hard.

- VENUS research reactor and Belgonucléaire fuel rod assembly plant in Belgium: two locations at the reactor, these being the ones where most occupational dose is likely to be accrued, and four positions at the fuel facility near fuel rods with differing amounts of shielding.

- Ringhals pressurised water reactor in Sweden: two locations within the reactor provided comparison with results from a previous measurement programme ${ }^{(7)}$ and two measurement locations around a spent fuel flask with its neutron shield in place.

- At a European nuclear facility where special nuclear material is stored: three positions around a storage facility were used, each with differing amounts of shielding. These measurements were needed because the neutron dose rate was high compared with the photon dose rate, and major modifications to the facility were planned.

\section{Reference dosimetry}

\section{Fluence-energy distribution measurements}

Energy distribution measurements were made by IRSN with their set of 12 multispheres ${ }^{(8)}$. Initial unfolding was via the program NUBAY, which uses a simple parameterisation of the spectrum based on three energy regions: a Maxwellian thermal distribution, an intermediate region that is flat in lethargy and a Maxwell fission distribution for the fast component. The solution energy distribution from NUBAY was used as one of the default energy distributions for the program GRAVEL, which is less constrained by the default energy distribution than NUBAY.

For all of the measurement locations, the NUBAY unfolding was confirmed by GRAVEL. In particular, the high energy peak was always at the same energy 


\section{EVALUATION OF INDIVIDUAL DOSIMETRY IN MIXED NEUTRON AND PHOTON FIELDS}

as the NUBAY solution: if it had not been, then the $H^{*}(10)$ rate would have had significant uncertainty.

These energy distributions provided reference values for $H^{*}(10)$ for the analysis of the survey instrument results. The output from the unfolding was used to give a measure of the uncertainty in the integral quantities. The values for $H^{*}(10)$ rate varied from over $2 \mathrm{mSv} \mathrm{h}^{-1}$ inside the storage facility in Campaign 4 to about $10 \mu \mathrm{Sv} \mathrm{h}^{-1}$ in the control room of the VENUS reactor.

\section{Directional spectrometry}

Determination of the direction distribution of the neutron field is necessary in order to calculate values for $H_{\mathrm{p}}(10)$ and effective dose. This is not easy because neutrons are detected via the secondary charged particles that they generate: the energy and direction of the secondary charged particles either has a weak correlation to the energy and direction of the neutron or, in some cases, no correlation to it.

Two systems were developed within the EVIDOS project. These contrasted significantly in approach.

- The DIMNP telescope-design directional neutron spectrometer ${ }^{(9)}$ uses superheated drop detectors at the centre of a $30-\mathrm{cm}$ diameter nylon-6 sphere. An aperture in the sphere ensures that the detector sees neutrons preferentially from one direction. Changing the temperature of the detector changes the threshold energy and rotating it changes the direction of maximum response. The bubbles are detected acoustically.

- The PTB directional spectrometer ${ }^{(10)}$ consists of six detector capsules mounted on a $30-\mathrm{cm}$ diameter polyethylene sphere. Each capsule contains a stack of four silicon detectors with different radiator/shield combinations that alter the energy dependence of response. The pulse height data of the silicon detectors are collected for subsequent analysis of the energy and direction distribution of the field.

Both systems require the energy and direction distribution of the field to be unfolded from the recorded data. The data from the PTB directional spectrometer were unfolded into 20 equal solid angle components that are defined by the faces of an icosahedron. The multispheres solution for the energy distribution was used as the starting point for the unfolding, which was performed using the code MAXED

An additional system was also developed within the EVIDOS project that attempted to determine $H_{\mathrm{p}}(10)$ via a halocarbon-12 sensor embedded in a $30 \times 30 \times 15 \mathrm{~cm}^{3}$ polymethyl methacrylate slab. This system, HpSLAB, bypasses the need to determine the full energy and direction distribution of the field.

\section{Personal dosemeters}

Three categories of personal dosemeter were used in the EVIDOS workplaces.

- Seven types of electronic dosemeter: Aloka PDM-313, PSI DIS-N, PTB DOS-2002, Synodys DMC 2000 GN, Saphymo Saphydose-n, Thermo Electron EPD-N and Thermo Electron EPD-N2.

- BTI-PND and BTI-BDT bubble detectors, both of which have many of the features of active dosemeters. The results from these detectors are presented separately and also as a combined value.

- Two etched-track dosemeters (PSI and HPA).

- Where convenient, the local dosemeters were also used.

\section{Neutron survey instruments}

Five different neutron survey instruments were used in almost all of the measurement locations: four commercially available moderator-type instruments (Studsvik 2202D, Berthold LB 6411, Harwell N91 and Thermo Electron WENDI-2) and a prototype based on a tissue-equivalent proportional counter (SSI Sievert Instrument). The readings on these instruments were of interest because the personal dosemeter results need to be related to the results of prospective radiation surveys.

\section{RESULTS}

The full results of the EVIDOS project have been summarised recently ${ }^{(11)}$. The first objective for each workplace was to obtain values for the reference rates for $H^{*}(10), H_{\mathrm{p}}(10)$ and effective dose. This was achieved via the multisphere system and the PTB directional spectrometer for most locations, although, for the simulated workplace fields CANEL and SIGMA and the VENUS reactor locations, MCNP calculations also played a very important role. The uncertainties on the energy and direction distribution measurements are inevitably greater than those on the energy distribution alone and, in practice, it was found that the multisphere results were needed to assist the unfolding of the PTB directional spectrometer data.

Significant interpolation of the fluence to doseequivalent conversion coefficients for effective dose and $H_{\mathrm{p}}(10)$ was required so that the 20 direction bins for the energy distribution could be applied. This was assisted by some new calculations of the personal dose equivalent in a slab phantom ${ }^{(12)}$, since the previously published data were insufficient for the purpose.

The measured and calculated dose rates for the workplaces are shown in Figure 1 as the ratios $E / H_{\mathrm{p}}(10)$ and $E / H^{*}(10)$. The data for the former 


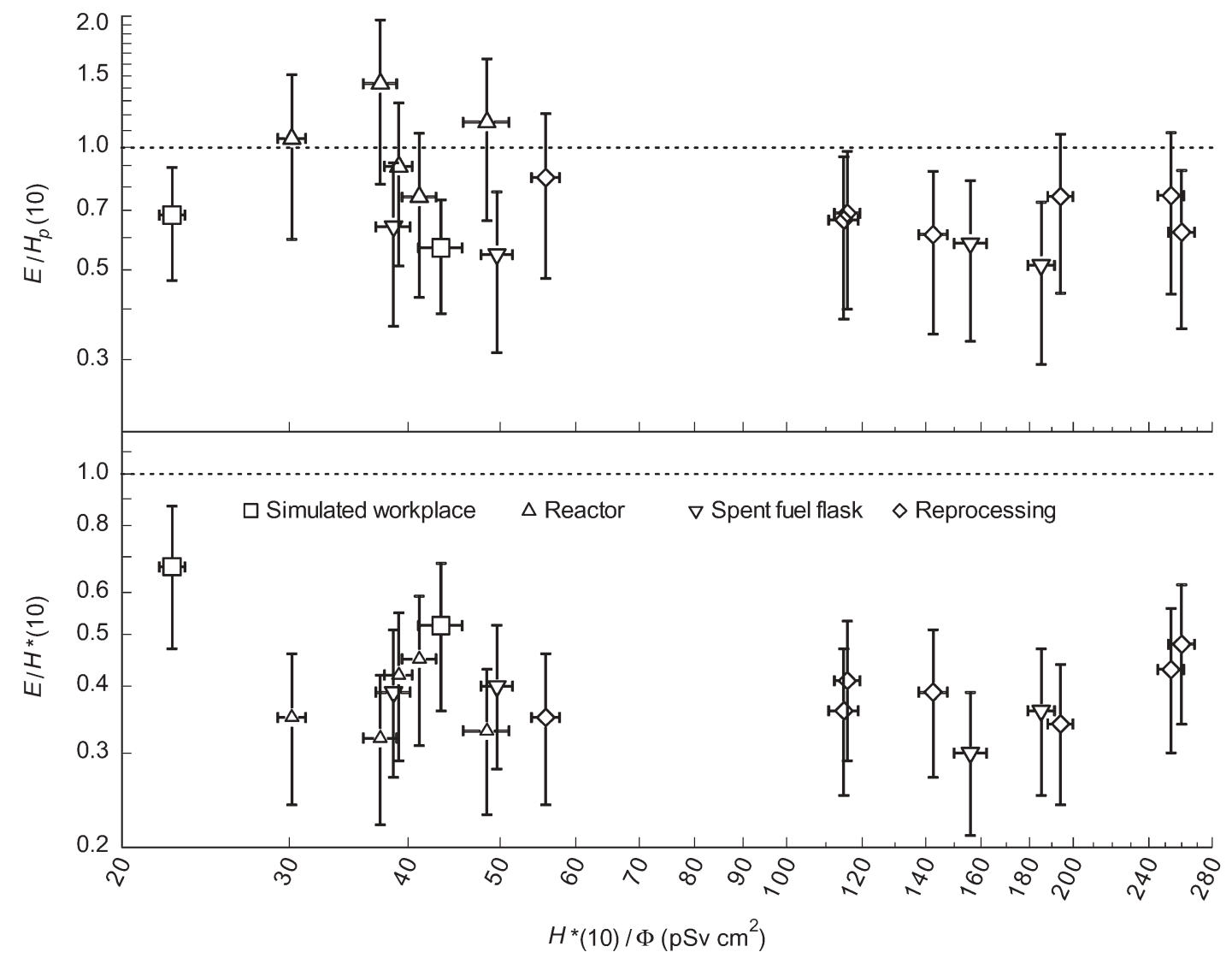

Figure 1. Ratios of the reference quantities determined during EVIDOS. The bottom part of the figure illustrates the ratio between the $E$ and $H^{*}(10)$ rates plotted against the average fluence to $H^{*}(10)$ conversion coefficient. The top part of the figure shows the ratio of $E$ to $H_{\mathrm{p}}(10)$ for the reference direction for the specific workplace.

show that effective dose is generally in the range from 50 to $80 \%$ of $H_{\mathrm{p}}(10)$. Consequently, for these fields an accurate assessment of $H_{\mathrm{p}}(10)$ will provide a conservative estimate of effective dose. However, significant underestimates of $H_{\mathrm{p}}(10)$ may result in underestimates of effective dose. The category of field for which this is least true is the group measured at nuclear reactors. In one case, the $H_{\mathrm{p}}(10)$ is barely $10 \%$ higher than the effective dose, and in three cases, it is lower. In the worst case, the effective dose is $42 \%$ higher than the $H_{\mathrm{p}}(10)$ : that field is the one measured inside the containment beneath the core of the reactor at the Krümmel BWR for which the dominant direction for the radiation is from above, so the use of a personal dosemeter on the front of the torso may not be appropriate. In all of these reactor fields, the importance of the scattered component of the field causes the $H_{\mathrm{p}}(10)$ to be comparable with or lower than the effective dose.
The data show that effective dose is generally only $30 \%$ to $50 \%$ of $H^{*}(10)$ (Figure 1 ). Consequently, there is a significant conservatism in-built for radiation surveys. The exception to this is the SIGMA simulated workplace field. That field has a very soft energy distribution but is not as isotropic as would be expected for such an energy distribution in the workplace.

The results of the personal dosemeters and survey instruments were reported using routine calibrations and algorithms. In some cases, the data that have been accumulated within the EVIDOS project have assisted the laboratories to enhance their procedures. All of the data were entered into a database, as the project progressed.

Significant overestimates and underestimates of $H_{\mathrm{p}}(10)$ were found in Figure 2. In this representation, the maximum and minimum responses are shown, although the minimum is omitted for those 


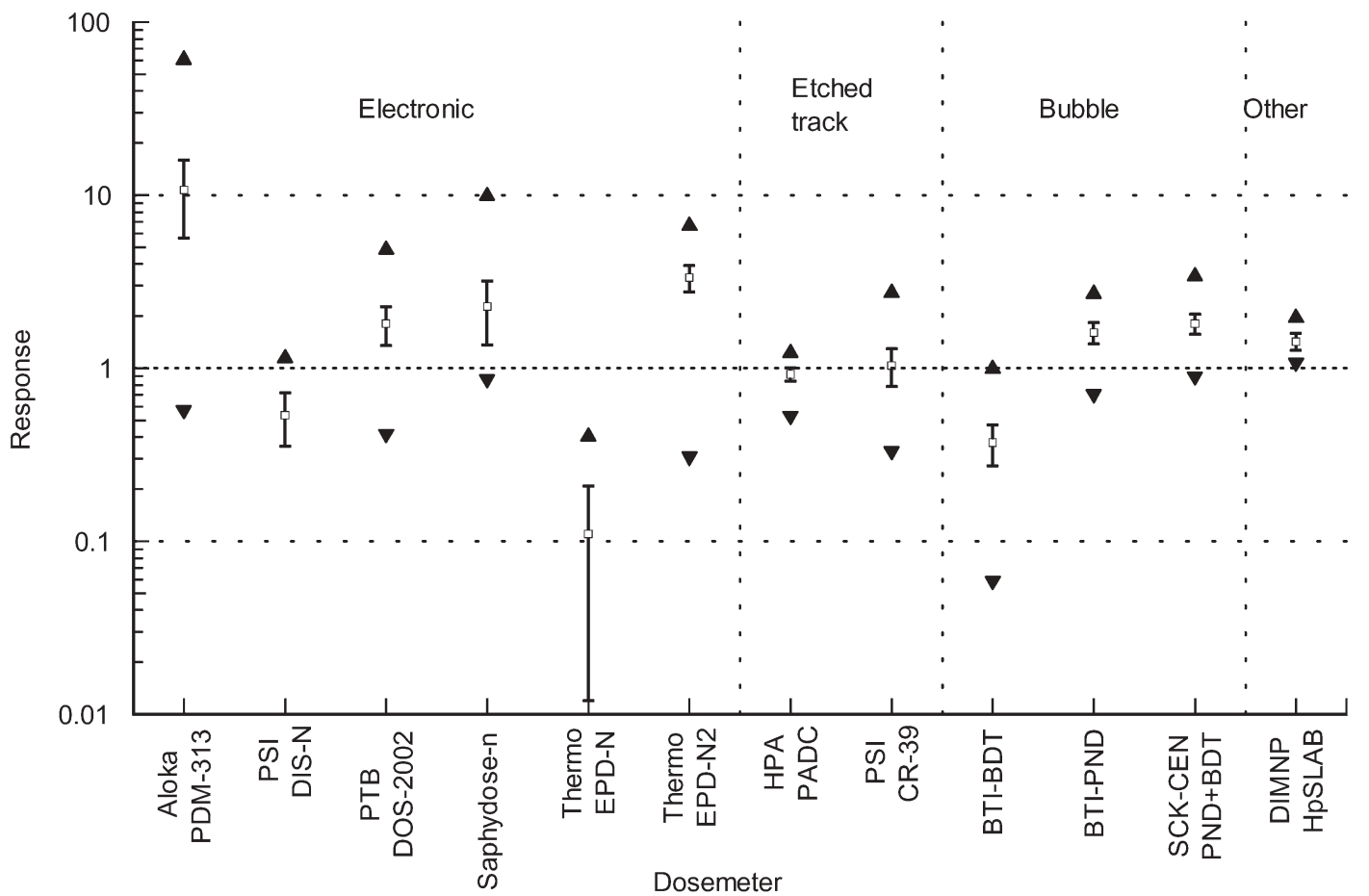

Figure 2. Responses of personal dosemeters in the EVIDOS project. The data are plotted as the mean value for all fields with uncertainties indicating the standard deviation. The extreme values are also indicated: $\boldsymbol{\Delta}$ shows the maximum value and $\boldsymbol{\nabla}$ the minimum value. Where no minimum value is indicated, the detection limit of the dosemeter caused the response to be zero.

fields where the detection level was not reached. Generally, it is seen that the personal dosemeters overestimate $H_{\mathrm{p}}(10)$ sometimes by large factors. However, systems that rely only on the detection of thermalised neutrons tend to underestimate the personal dose equivalent, sometimes very significantly.

The potential for overestimates of $H_{\mathrm{p}}(10)$ is greatest with the electronic dosemeters, except where the dosemeter has no significant fast neutron response, whereas the passive systems show the least potential for bias. Most of the personal dosemeters have the potential for underestimating by more than a factor of two (Figure 2), which will cause underestimates of effective dose in some fields. When a personal dosemeter underestimates in one of the fields for which the effective dose is larger than the personal dose equivalent, then the assessment of the personal dose equivalent may be much lower than the effective dose.

The assessments of the $H^{*}(10)$ made using moderator type survey instruments were never more than $30 \%$ lower than the reference value obtained using the multispheres. Given the relationship between effective dose and $H^{*}(10)$ for the fields in this study (Figure 1), the underestimates of effective dose can be ruled out. A greater concern with these instruments may be excessive overestimation of effective dose, since the assessment of $H^{*}(10)$ in some soft fields can give a factor of five overestimate of effective dose. In some of the softest fields, the TEPCbased survey instrument produced underestimates of $H^{*}(10)$ of more than a factor of two.

\section{CONCLUSIONS}

The ability to determine the energy and direction distribution of the field in workplaces developed within the EVIDOS project has enabled the performance of survey instruments and personal dosemeters to be qualified more accurately in the circumstances in which they are used. This is a significant and complementary step forward, because the conditions in the workplace are the ones that are truly relevant, and they are in some respects more demanding than those encountered in the calibration laboratory.

The relationships between the dose quantities are themselves interesting, since they have implications for the assessment of risk via radiation surveys and personal dosemeter readings. In the fields used in 


\section{R. J. TANNER ET AL.}

this project, the ambient dose equivalent is, in general, more than twice as large as the effective dose, which effectively rules out the underestimates of effective dose rates in radiation surveys. However, the assessment of effective dose could, in some circumstances, provide significant overestimates of ambient dose equivalent.

For personal dosemeters, the analysis is significantly more complex. The personal dose equivalent is rarely much smaller than the effective dose, which means that the underestimates of personal dose equivalent will potentially provide the underestimates of effective dose. In some instances, the effective dose rate has been found to be higher than the personal dose equivalent rate. In these circumstances, the location of the dosemeter on the body may be a significant consideration: in the most extreme case in this work, where the primary direction of the neutrons is from above, dosemeters worn on the front of the torso do not perform adequately.

In terms of simple dosimetric performance, the electronic dosemeters do not yet perform as well as etched track detectors or bubble detectors. They have advantages because they are active devices, but the ones included in this study are prone to significant overestimates of the personal dose equivalent and, in some cases, significant underestimates. It must be noted that these devices are relatively new, and it may hence be expected that this performance will improve with time. Indeed, some of the designs used have improved during the measurement programme.

The main achievements of the EVIDOS project lie in the field characterisation. Previously, determination of $H_{\mathrm{p}}(10)$ and effective dose rates in the workplace has tended to rely on assumptions about field geometry, whereas, in this work, the assessment of the performance of personal dosemeters has been made possible through the simultaneous determination of the energy and direction distributions of the fields.

\section{ACKNOWLEDGEMENTS}

The Nuclear Fission Safety programme of the European Commission funded this work. Additional support staff at the participating laboratories provided valuable input to the project as did health physics staff at the laboratories where the measurements were made.

\section{REFERENCES}

1. Alberts, W. G., Dietz, E., Guldbakke, S. and Kluge, H. Response of an electronic personal dosemeter. Radiat. Prot. Dosim. 51, 207-210 (1994).

2. Alberts, W. G. et al. Advanced methods of active neutron dosimetry for individual monitoring and radiation field analysis (ANDO). PTB-Bericht N39 (Braunschweig: PTB) (1999).

3. Thomas, D. J. and Klein, H. (eds). A handbook on neutron and photon spectrometry techniques for radiation protection. Radiat. Prot. Dosim. 107 (1-3) (2003).

4. d'Errico, F., Bartlett, D. T., Ambrosi, P. and Burgess, PDetermination of direction and energy distributions. Radiat. Prot. Dosim. 107, 133-153 (2003).

5. Gressier, V. et al. Characterisation of the IRSN CANEL/T400 facility producing realistic neutron fields for calibration and test purposes. Radiat. Prot. Dosim. 110, 523-527 (2004).

6. Lacoste, V., Gressier, V., Muller, H. and Lebreton, L. Characterisation of the IRSN graphite moderated americium-beryllium neutron field. Radiat. Prot. Dosim. 110, 135-139 (2004).

7. Lindborg, L., Bartlett, D. T., Drake, P., Klein, H., Schmitz, Th. and Tichy, M. Determination of neutron and photon dose equivalent at workplaces in nuclear facilities in Sweden (A joint SSI-EURADOS comparison exercise). Radiat. Prot. Dosim. 61, 89-100 (1995).

8. Lacoste, V., Reginatto, M., Asselineau, B. and Muller, $\mathrm{H}$. Bonner sphere neutron spectrometry at nuclear workplaces in the framework of the EVIDOS project. Radiat. Prot. Dosim. 2007, 27 February (Epub ahead of print).

9. d'Errico, F., Giusti, V., Reginatto, M. and Wiegel, B. $A$ telescope-design directional neutron spectrometer. Radiat. Prot. Dosim. 110 (1-4), 533-537 (2004).

10. Luszik-Bhadra, M., Reginatto, M. and Lacoste, V. Measurement of energy and direction distribution of neutron and photon fluences in workplace fields. Radiat. Prot. Dosim. 110, 237-241 (2004).

11. Schuhmacher, H. et al. Evaluation of individual dosimetry in mixed neutron and photon radiation fields. PTB report PTB-N-49 (Braunschweig: PTB) (2006).

12. d'Errico, F., Giusti, V. and Siebert, B. R. L. A new neutron monitor and extended conversion coefficients for $H_{p}(10)$. To be published in Radiat. Prot. Dosim. 